\title{
Manual acupuncture for the infertile female with polycystic ovary syndrome (PCOS): study protocol for a randomized sham- controlled trial
}

Qiao Wang ${ }^{1}$, Haiping Deng ${ }^{1}$, Ke Cheng ${ }^{1}$, Zouqin Huang ${ }^{2}$, Xiuqi Yin ${ }^{3}$, Yichen Zhou ${ }^{3}$, Yiqin Yang ${ }^{3}$, Weidong Shen ${ }^{3}$, Ling Zhao ${ }^{1 *}$ and Xueyong Shen ${ }^{1 *}$ (D)

\begin{abstract}
Background: Polycystic ovary syndrome (PCOS) is one of the most common endocrine diseases for women. Acupuncture is widely used for the infertile female because of it is non-invasive and has fewer side effects, but the powerful evidence for the clinic is still insufficient. Our study intends to explore the effect of manual acupuncture (MA) in the infertile female with PCOS.

Methods: This study is a randomized, sham-controlled, patient-and assessor-blinded trial and aims to evaluate the effect of MA in women with PCOS and infertility. We will recruit 86 women aged 20-40 years with a diagnosis of infertility with PCOS. Participants will be randomly allocated in a 1:1 ratio to the MA group and the sham acupuncture (SA) group. Both groups will receive real herbal medicine treatment as a basic treatment twice a day for three menstrual cycles, the MA group receive real acupuncture treatment and the SA group received placebo acupuncture treatment (non-penetrating). All patients will receive acupuncture treatment twice per week for three menstrual cycles. The primary outcome is pregnancy rate and secondary outcomes include ovulation rate, sex hormones, insulin resistance index (IRI), PCOS symptoms, and Traditional Chinese Medicine (TCM) syndrome scores. Outcome measures will be collected at baseline, each menstrual cycle, the end of treatments, and six months after the last acupuncture treatment. The present protocol followed the SPIRIT guidelines and fulfilled the SPIRIT checklist.
\end{abstract}

Discussion: This study will be conducted to compare the efficacy of MA versus SA. This trial will help to evaluate whether MA is effective in increasing pregnancy and ovulation rates of the infertile female with polycystic ovary syndrome.

Trial registration: Chinese Clinical Trial Registry, ChiCTR1800014997. Registered on 27 February 2018.

Keywords: Polycystic ovary syndrome, Manual acupuncture, Traditional Chinese Medicine, Infertility, Herb medicine

\footnotetext{
* Correspondence: zhao3helen@sina.com; sxy1@shutcm.edu.cn

'Acupuncture \& Tuina School, Shanghai University of Traditional Chinese

Medicine, Shanghai 201203, China

Full list of author information is available at the end of the article
}

(C) The Author(s). 2019 Open Access This article is distributed under the terms of the Creative Commons Attribution 4.0 International License (http://creativecommons.org/licenses/by/4.0/), which permits unrestricted use, distribution, and reproduction in any medium, provided you give appropriate credit to the original author(s) and the source, provide a link to the Creative Commons license, and indicate if changes were made. The Creative Commons Public Domain Dedication waiver (http://creativecommons.org/publicdomain/zero/1.0/) applies to the data made available in this article, unless otherwise stated. 


\section{Background}

Polycystic ovary syndrome (PCOS) is one of the most common endocrine diseases for women. Diagnosis is based on two of the following three findings: (1) oligoand/or anovulation; (2) clinical and/or biochemical signs of hyperandrogenism; and (3) polycystic ovaries and exclusion of other etiologies [1]. The prevalence of PCOS under the Rotterdam criteria was $19.9 \%$ in 392 women aged 18-45 years [2]. It is one of the main causes of infertility in women during the reproductive period. PCOS causes $>75 \%$ of causes of anovulatory infertility [3].

Clomiphene citrate $(\mathrm{CC})$ is the first-line treatment in anovulatory subfertility patients with PCOS. Gonadotrophins such as follicle-stimulating hormone (FSH) are, in addition to surgery, second-line treatments [4]. The cumulative ovulation rate of CC is as high as $90 \%$, with a pregnancy rate of $50-70 \%$, but the multiple pregnancy rate is up to $15 \%$ and rate of ovarian hyper-stimulation syndrome (OHSS) is $2 \%$ [5]. Meanwhile, it also has other impact effects [6] such as reversible ovarian enlargement ( $>10 \%)$, visual symptoms, headaches, etc. Some studies $[7,8]$ reported that there were several associations between use of CC and birth defects.

Acupuncture and Traditional Chinese Medicine (TCM) methods have a long history of use for gynecological diseases [9] and the related research has increased in the past 20 years [10]. A prevalence study [11] reported that $29 \%$ of 428 infertile couples in eight community and academic infertility practices had used a complementary and alternative medicine (CAM) treatment for infertility, $22 \%$ had tried acupuncture, and $17 \%$ had tried herbal therapy. A review [12] investigated whether utility of TCM would be a feasible method to improve the outcome of female infertility treatment because of inadequate evidence. A meta-analysis [13] demonstrated that co-treatment with TCM and letrozole was more effective than letrozole monotherapy in the treatment of PCOS. Yanjing et al. [14] found that Chinese herbal formula combined with Electroacupuncture (EA) can remarkably improve the menstrual cycle, reduces body weight and the levels of luteinizing hormone (LH), LH/ follicle-stimulating hormone (FSH), total testosterone $(\mathrm{T})$, anti-Mullerian hormone $(\mathrm{AMH})$, improve ovulation, and pregnancy rates. We therefore selected Chinese herbal medicine as a basic treatment.

Recently, a scoping review [15] found that acupuncture had the highest level of evidence for its use among 12 different CAM methods in improving fertility outcomes although this evidence is inconclusive. Some evidence $[16,17]$ indicated that acupuncture was probably an effective treatments for PCOS, which can improve patients' clinical symptoms, sex hormone levels, and menstrual cycle and effectively shorten reproduction cycles in infertility patients with PCOS. A systematic review [18] showed that acupuncture can be used to regulate menstruation, assist conception in women, and not increase the risk of multiple pregnancies, but the evidence is insufficient. There is a close relationship between the arrival of qi and therapeutic outcome. As The Yellow Emperor's Inner Classic: The Spiritual Pivot-The Nine Kinds of Needles and the Twelve Source Points [19] states: "The most important thing in acupuncture is to get the acupuncture feeling, when it appears, the curative effect will appear." We therefore use manual acupuncture (MA) to obtain the qi sensation (acupuncture feeling) to get the curative effect.

Nevertheless, Pastore et al. [20] also claimed that both true acupuncture (TA) and sham acupuncture (SA) had a similar improvement in their LH/FSH ratio for these women with PCOS. A randomized clinical trial [21] did not support acupuncture as an infertility treatment in such women. The conclusion of the multicenter-large sample study [22] was potentially incorrect because it had serious flaws. Indeed, the evidence for the effect of acupuncture in the treatment for infertile women with PCOS is still insufficient. Therefore, this study aims to investigate the clinical effectiveness of acupuncture in infertile women with PCOS. Our hypothesis is that MA will regulate the menstrual cycle and improve the ovulation and pregnancy rates.

\section{Methods/design}

\section{Study design}

This is a randomized, sham-controlled, patient-and assessor-blinded trial. A target sample of 86 participants will be recruited from the gynecology clinic at Shuguang Hospital. The flow chart is shown in Fig. 1. The present protocol followed the SPIRIT guidelines and fulfilled the SPIRIT checklist (Additional file 1). The trial protocol is in accordance with the principles of the Declaration of Helsinki and has been approved by Institution review board (IRB) of Shuguang Hospital (the Affiliated Hospital of Shanghai University of TCM) (approval no. 2017-569-52-01). This trial was registered at the Chinese Clinical Trial Registry (ChiCTR1800014997). Any changes that need to be made in the trial protocol will be communicated to all investigators, the ethics committees, and the trial registry. Written informed consent will be obtained from each participant.

\section{Sample size, randomization, and blinding}

Due to the lack of sufficient preliminary studies, it is difficult to determine adequate sample size. According to the studies [23, 24], we hypothesize that the pregnancy rate after MA is $76 \%$ and the pregnancy rate after SA is $40 \%$. The calculation equation we used was $\alpha=0.05,1$ $\beta=0.80$ and we determined that a sample size of $36 \mathrm{pa}-$ tients in each group would be sufficient to detect the statistical difference between the two groups, allowing for a $20 \%$ withdrawal rate. We will plan to enroll a total 


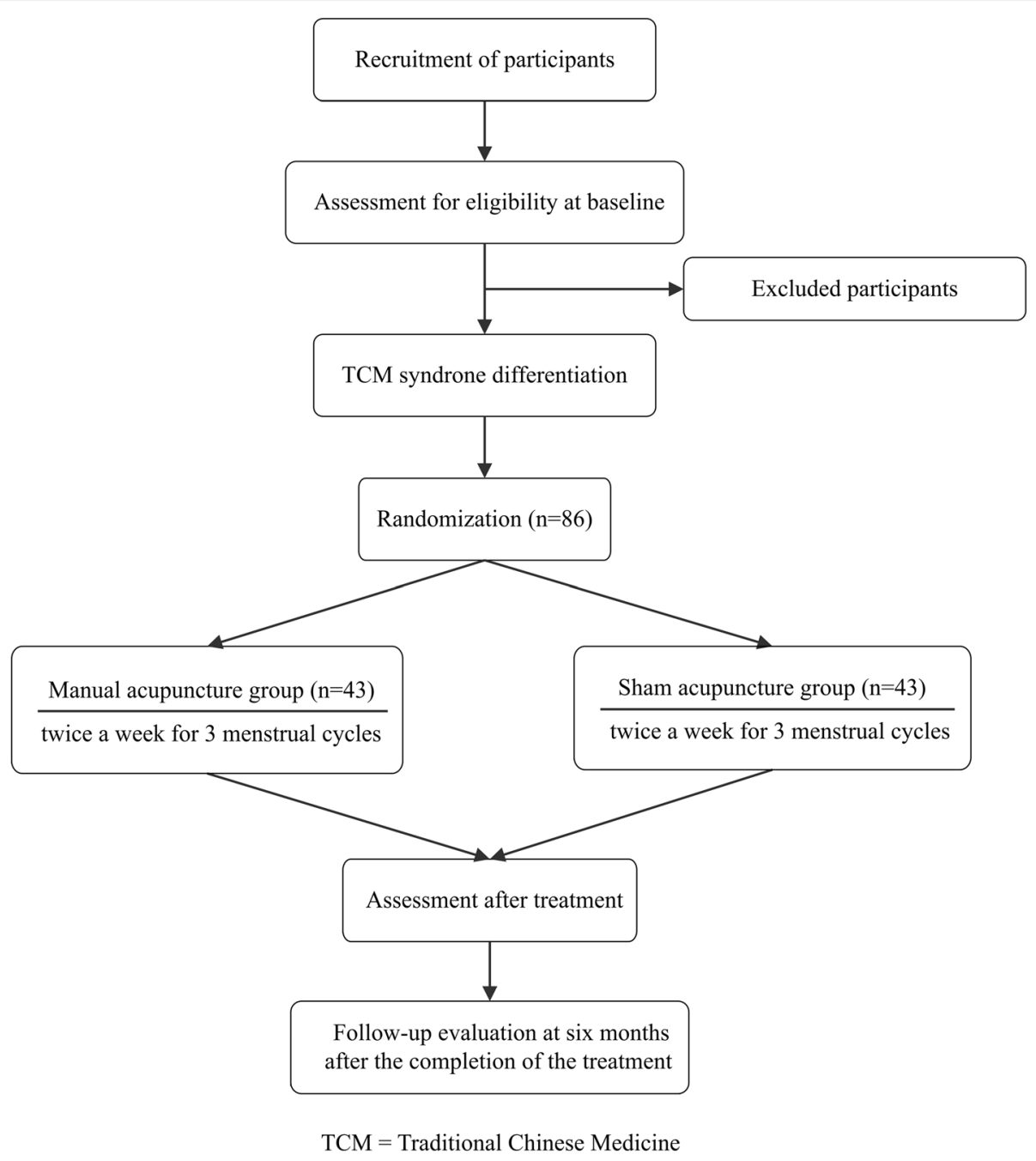

Fig. 1 Study flow chart

of 86 participants with 43 participants in each group. According to the random number sequence generated by Excel, the patients will be randomly divided into either the MA group or the SA group in a 1:1 ratio. We set the blind code in the case for remaining blind when the patients have the adverse effects. The random code and blind code will be conducted by a "third party" independent of the study using opaque envelopes. The envelopes will be sealed and shuffled and the assignment records will not be disclosed until the end of the study. Trial participants, formula doctor, outcome assessors, and data analysts will be blinded to the treatment allocation to minimize potential sources of bias. Because of the special nature of acupuncture, only acupuncturists will not be blinded to the treatment allocation.

\section{Inclusion criteria}

Participants who meet all the following requirements will be allowed for enrollment:
1. Women aged $20-40$ years.

2. Patients who meet the diagnostic criteria for both infertility and PCOS:

a) Patients who meet the diagnosis of PCOS defined by the Rotterdam criteria proposed in 2003 [1]:

- Oligomenorrhea or amenorrhea: oligomenorrhoea is defined as an intermenstrual interval $>35$ days or $<8$ menstrual bleedings in the past year. Amenorrhea is defined as complete cessation of menstrual cycles for six months or more when a patient has previously had regular cycles and for 12 months or more when the patient has had irregular cycles;

- Clinical or biochemical hyperandrogenism: biochemical hyperandrogenemia is defined as a total serum testosterone concentration > $60 \mathrm{ng} / \mathrm{dL}$ and clinical hyperandrogenism is 
defined as a Ferriman-Gallwey (FG) score $\geq 5$ in mainland China;

- Polycystic ovary morphology: this is defined as $\geq 12$ antral follicles (2-9 $\mathrm{mm}$ in diameter) or an ovarian volume $>10 \mathrm{~mL}$ on transvaginal scanning;

b) Patients who meet the diagnosis of infertility [25]: women who fail to achieve a clinical pregnancy after 12 months or more of regular unprotected sexual intercourse; this includes secondary infertility which is failure to conceive after a previous pregnancy.

3. Husband's semen examination is normal.

4. Willingness to sign the consent form.

\section{Exclusion criteria}

Participants meeting any of the following criteria will be excluded:

1. Hyperprolactinemia, adrenal or ovarian tumors secreting abnormal increase of androgen, thyroid disease, suspected Cushing syndrome, and other heart and kidney diseases.

2. Those who had used hormone drugs such as estrogen or oral contraceptives in the last month.

3. Those who had used other drugs that affect reproductive function or metabolism (such as antiobesity drugs, anti-diabetic drugs, etc.) or have participated in other clinical trials in the past two months.

4. Those who had received acupuncture treatment.

5. Those who had other infertility factors (such as tubal blockage, immune infertility, etc.).

6. Those who were unable to cooperate with the completion of the study, including patients with infectious diseases, mental illness, and other medical histories.

\section{Interventions}

Both TA and SA groups will receive acupuncture sessions for a total of three menstrual cycles. All the patients will receive herbal medicine twice a day for three menstrual cycles. The same doctor (the professor of obstetrics and gynecology in Shuguang hospital who has 35 years of work experience) will slightly adjust the formula each week depending on the changes in the patients' symptoms, tongue coating, and pulse (main herbal formula components are shown in Table 1). During the menstrual period, patients will be orally administered the Taohong Siwu decoction. Non-menstrual period treatment will be based on pattern differentiation: (1) spleen-kidney Yang deficiency will be given Bushen Tiaojing decoction; and (2) liver-kidney Yin deficiency will be given Guishao Dihuang decoction. Participants will receive all herbs from the same hospital and will be instructed on how to make the decoction.

According to the textbook Acupuncture and Moxibustion [26], and taking opinions from Chinese acupuncture experts, the essential acupuncture points selected are as follows: RN4 (Guanyuan); EX-CA1 (Zigong); ST29 (Guilai); ST36 (Zusanli); and SP6 (Sanyinjiao). The additional individualized acupuncture points will be chosen by the practitioners according to the patterns of identification: (1) spleen-kidney Yang deficiency plus RN6 (Qihai), RN12 (Zhongwan), ST25 (Tianshu); and (2) liver-kidney Yin deficiency will add with bilateral KI3 (Taixi), KI6 (Zhaohai). According to the menstrual cycle, the follicular phase will use the acupuncture points bilateral KI3 (Taixi) and KI6 (Zhaohai); the ovulatory phase will use the acupuncture points bilateral LR3 (Taichong), SP10 (Xuehai), PC6 (Neiguan), and DU20 (Baihui) (Table 2). A placebo device [27] will be applied in both groups for better implementation of blindness. The schematic diagram of acupuncture treatment is shown in Fig. 2. The acupuncture treatment in both groups will take 30 min per session, twice per week, from the third day of menstruation to the third day after ovulation for three menstrual cycles. Acupuncture treatments will be stopped upon a positive pregnancy test. The test will be done on the 14th day after each ovulation.

\section{Manual acupuncture group}

Disposable, stainless steel needles $(0.25 \times 40 \mathrm{~mm}$, provided by Wuxi Jiajian Medical Instrument Co. Ltd., China) will be punctured through the device when patients are in the supine position and inserted $10-30 \mathrm{~mm}$ deep, depending on location. The needles will be retained for $30 \mathrm{~min}$ and manually lifted, thrust, twisted, and rotated moderately every $10 \mathrm{~min}$ to maintain the deqi sensation. When qi arrives, the acupuncturist will feel tightness around the needle, the patients will feel such sensations as soreness, numbness, distention, and heaviness at the point. These sensations can radiate along the course of the channel.

\section{Sham acupuncture group}

The procedure and duration of treatment in the SA group will be identical in the TA group except the needles $(0.25 \times 25 \mathrm{~mm}$, blunt tip, provided by Wuxi Jiajian Medical Instrument Co. Ltd., China) are blunt tip and there will be no skin penetration or manual stimulation.

\section{Practitioner background}

The TA and SA treatments will be conducted by acupuncturists who are licensed by the Chinese medicine practitioner qualification certificate and who specialize in acupuncture with at least three years of clinic experience. They will have studied acupuncture for $>10$ years and graduated from a TCM university. All practitioners will undergo intensive and customized 
Table 1 Main herbal formula selection

\begin{tabular}{llll}
\hline Period & Pattern & Formula & Composition \\
\hline $\begin{array}{l}\text { Menstrual } \\
\text { period }\end{array}$ & $\begin{array}{l}\text { Taohong Siwu } \\
\text { decoction }\end{array}$ & $\begin{array}{l}\text { Radix Rehmanniae Preparata, Radix Angelicae Sinensis, Radix Paeoniae Alba, Rhizoma } \\
\text { Chuanxiong, Semen Persicae, Flos Carthami }\end{array}$ \\
$\begin{array}{l}\text { Non- } \\
\text { menstrual } \\
\text { period }\end{array}$ & $\begin{array}{l}\text { Spleen-kidney } \\
\text { Yang deficiency }\end{array}$ & $\begin{array}{l}\text { Bushen Tiaojing } \\
\text { decoction }\end{array}$ & $\begin{array}{l}\text { Radix Angelicae Sinensis, Rhizoma Chuanxiong, Radix Rehmanniae Preparata, Radix Paeoniae } \\
\text { Alba, Poria, Radix Salviae Miltiorrhizae, Semen Cuscutae, Radix Astragali, Fructus Lycii, Rhizoma } \\
\text { Dioscoreae }\end{array}$ \\
& $\begin{array}{ll}\text { Liver-kidney Yin } \\
\text { deficiency }\end{array}$ & $\begin{array}{l}\text { Guishao Dihuang } \\
\text { decoction }\end{array}$ & $\begin{array}{l}\text { Radix Rehmanniae, Radix Paeoniae Alba, Fructus Lycii, Cortex Moutan, Rhizoma Anemarrhenae, } \\
\text { Radix Ginseng, Radix Glycyrrhizae, Cortex Lycii }\end{array}$ \\
& &
\end{tabular}

training for a full understanding of the SA procedure and will be trained to administer acupuncture using a sham needle device. The techniques for the entire treatment procedure will be standardized between practitioners.

\section{Outcome measures}

Outcomes will be collected at baseline, each menstruation, after three menstrual cycles of treatment, and follow-up at six months after the completion of the treatment. The overview of the outcome measurement at the different time points is shown in Table 3.

\section{Primary outcome}

The primary outcome is the pregnancy rate during the trial, including ectopic pregnancy. Blood $\beta$-HCG will be measured at 14 days after ovulation.

\section{Secondary outcome}

Ovulation rate Ovulation will be observed through Basal body temperature (BBT) or B ultrasound every

Table 2 Acupuncture point selection

\begin{tabular}{|c|c|}
\hline Point & Location \\
\hline $\begin{array}{l}\text { RN4 } \\
\text { (Guanyuan) }\end{array}$ & On the lower abdomen at the anterior midline, 3 cun below the umbilicus \\
\hline $\begin{array}{l}\mathrm{EX}-\mathrm{CA} 1^{\mathrm{a}} \\
\text { (Zigong) }\end{array}$ & On the lower abdomen, 4 cun below the center of the umbilicus and 3 cun lateral to the anterior midline \\
\hline ST29a (Guilai) & 1 cun cranial to the pubic bone and 2 cun lateral of the midline \\
\hline ST36 ${ }^{\mathrm{a}}$ (Zusanli) & At the anterior aspect of the leg 3 cun inferior to ST35 (Dubi) on the line connecting ST35 (Dubi) to ST41 (Jiexi) \\
\hline $\begin{array}{l}\mathrm{SPG}^{\mathrm{a}} \\
\text { (Sanyinjiao) }\end{array}$ & $\begin{array}{l}\text { On the tibial aspect of the leg posterior to the medial border of the tibia, } 3 \text { cun superior to the prominence of the medial } \\
\text { malleolus }\end{array}$ \\
\hline \multicolumn{2}{|l|}{ Syndrome 1} \\
\hline RN6 (Qihai) & With the patient supine, the point is halfway between the center of the umbilicus and RN4 (Guan yuan) \\
\hline $\begin{array}{l}\text { RN12 } \\
\text { (Zhongwan) }\end{array}$ & On the upper abdomen at the anterior median line, 4 cun above the umbilicus \\
\hline $\mathrm{ST} 25^{\mathrm{a}}$ (Tianshu) & On the upper abdomen 2 cun lateral to the center of the umbilicus \\
\hline \multicolumn{2}{|c|}{ Syndrome 2 and phase 1} \\
\hline $\mathrm{KI} 3^{\mathrm{a}}$ (Taixi) & $\begin{array}{l}\text { On the posteromedial aspect of the ankle in the depression between the prominence of the medial malleolus and the calcaneal } \\
\text { tendon }\end{array}$ \\
\hline $\mathrm{KI}^{\mathrm{a}}$ (Zhaohai) & $\begin{array}{l}\text { On the medial aspect of the foot in the depression inferior to the medial malleolus, } 1 \text { cun inferior to the prominence of the medial } \\
\text { malleolus }\end{array}$ \\
\hline \multicolumn{2}{|l|}{ Phase 2} \\
\hline LV3 ${ }^{\mathrm{a}}$ (Taichong) & $\begin{array}{l}\text { On the dorsum of the foot between the first and second metatarsal bones in the depression distal to the junction of the bases of } \\
\text { the two bones and over the dorsalis pedis artery }\end{array}$ \\
\hline SP10a (Xuehai) & $\begin{array}{l}\text { With the knee flexed, on the medial side of the thigh } 2 \text { cun above the superior medial corner of the patella on the prominence of } \\
\text { the medial head of the quadriceps muscle of the thigh }\end{array}$ \\
\hline PC6 ${ }^{\mathrm{a}}$ (Neiguan) & $\begin{array}{l}\text { On the medial aspect of the forearm between the palmaris longus and the flexor carpi radialis tendons, } 2 \text { cun proximal to the } \\
\text { palmar wrist crease }\end{array}$ \\
\hline DU20 (Baihui) & On the head at the anterior median line, 5 cun superior to the anterior hairline \\
\hline
\end{tabular}

Based on pattern and phase differentiation, syndrome 1 = Spleen-kidney Yang deficiency; syndrome 2 = Liver-kidney Yin deficiency, phase 1 = follicular phase; phase 2 = ovulatory phase

ailateral points 


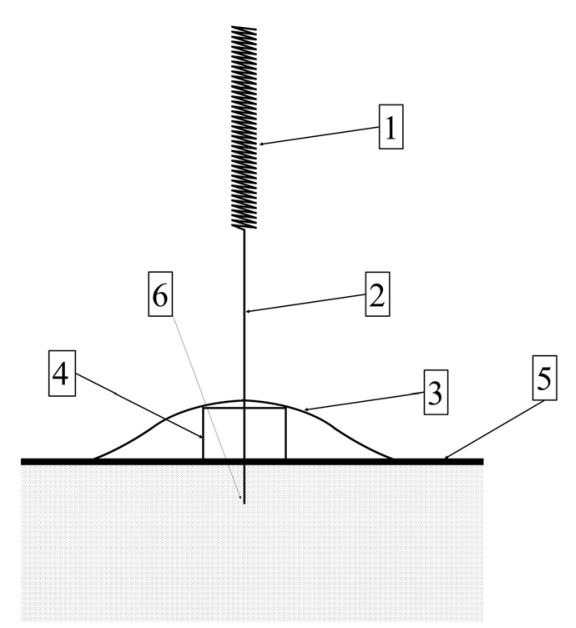

Manual acupuncture

1.needle handle 2.needle body 3 . desensitized

transparent tape 4.adhesive pad 5.cutis 6.sharp tip

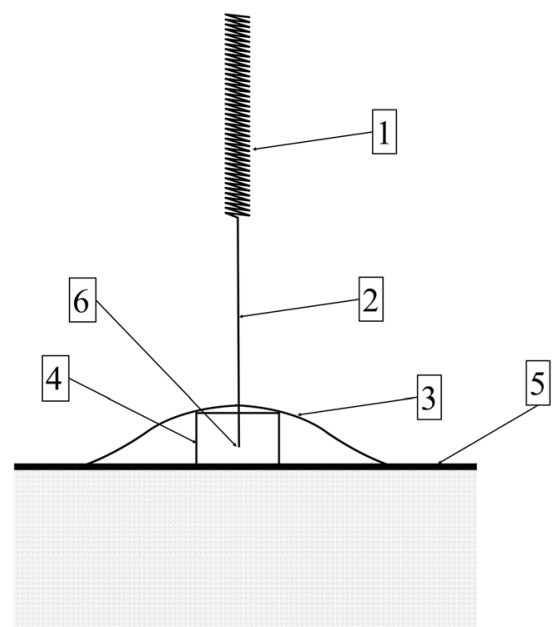

Sham acupuncture

1.needle handle 2 .needle body 3 . desensitized

transparent tape 4.adhesive pad 5.cutis 6.blunt tip

Fig. 2 Schematic diagram of acupuncture device

menstrual cycle. From the 10th day of the menstrual period, the patients will receive $B$ ultrasound 3-4 times to observe the size of the follicle and whether it has discharged.

Syndrome scores The PCOS syndrome scores will be recorded before and after treatment to assess whether the treatment improved the patient's signs of PCOS.

The TCM syndrome scores will be recorded by the same professor once a week to adjust the acupuncture and herbal treatment [28] and evaluate the improvement of the patients' TCM syndromes.
Blinding assessment Assessment for patients' blinding was conducted after the last treatment session. All patients will be asked to guess whether they have received TA or SA.

Sex hormones and IRI The fasting blood samples will be drawing in the third day of menstruation before and after treatment by the nurse. The chemiluminescence immunoassay (CLIA) will be used to determine serum sex hormone levels include estradiol (E2), T, progesterone (P), FSH, LH, prolactin (PRL), dehydroepiandrosterone sulfate (DHEAS), sex hormone-binding globulin

Table 3 Overview of study visits

\begin{tabular}{|c|c|c|c|c|c|}
\hline & \multirow{2}{*}{$\begin{array}{l}\text { Screening } \\
\text { and } \\
\text { baseline } \\
\text { visit }\end{array}$} & \multicolumn{3}{|c|}{ Menstrual cycles } & \multirow{2}{*}{$\begin{array}{l}\text { Six months } \\
\text { after the last } \\
\text { acupuncture } \\
\text { treatment }\end{array}$} \\
\hline & & $1 s t$ & 2nd & $3 r d$ & \\
\hline General condition $^{\text {a }}$ & $\sqrt{ }$ & & & & \\
\hline Gynecological examination & $\sqrt{ }$ & & & & \\
\hline Fasting blood samples for sex hormone ${ }^{b}$, FINS, FBG, HbA1c & $\sqrt{ }$ & & & $\sqrt{ }$ & \\
\hline Transvaginal ultrasound & $\sqrt{ }$ & $\sqrt{ }$ & $\sqrt{ }$ & $\sqrt{ }$ & \\
\hline Pregnancy test (blood $\beta$-HCG) & & & & $\sqrt{ }$ & \\
\hline Treatment record $^{c}$ & & $\sqrt{ }$ & $\sqrt{ }$ & $\sqrt{ }$ & \\
\hline Questionnaire $^{d}$ & $\sqrt{ }$ & & & $\sqrt{ }$ & \\
\hline Telephone follow-up ${ }^{e}$ & & & & & $\sqrt{ }$ \\
\hline
\end{tabular}

${ }^{\mathrm{a}}$ General condition include age, $\mathrm{BMI}$, menstrual and obstetrical histories

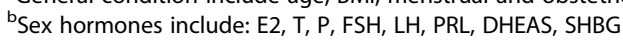

'Treatment record includes date and adverse events

${ }^{\mathrm{d}}$ Questionnaire includes the PCOS and TCM syndrome scores

eInquires if the participants were pregnant

E2 estradiol, $T$ total testosterone, $P$ progesterone, $F S H$ follicle-stimulating hormone, $L H$ luteinizing hormone, $P R L$ prolactin, $D H E A S$ dehydroepiandrosterone sulfate, $S H B G$ sex hormone-binding globulin, BMI body mass index, FINS fasting insulin, FBG fasting blood-glucose, HbA1C glycated hemoglobin 
(SHBG), and fasting serum insulin (Model: Switzerland ELECSYS2011). The oxidase method will be used to measure fasting plasma glucose through automatic biochemical analyzer (Model: Japan OLMPUS AU 400). These tests will all performed by the Shuguang Hospital Medical Testing Center.

The gold standard for investigating and quantifying insulin resistance is the "hyperinsulinemic euglycemic clamp" [29], but it rarely performed in clinical because of the invasive. The insulin resistance index (HOMA-IR) is a simpler test and is calculated by the equation (HOMA$\mathrm{IR}=$ fasting blood glucose $\times$ fasting insulin/22.5).

Follow-up Six months after the completion of the treatment, follow-up will be performed by telephone to ask if the participants were pregnant.

\section{Adverse events/serious adverse events}

We will monitor adverse events for each treatment during the trial, including hematomas and acute pain. Any adverse events or reactions that are thought to be causally associated with the intervention will be recorded, managed, and reported to the study coordinators. Serious adverse reactions will be reported to the ethical committee.

\section{Data analysis}

All statistical analyses of the data will be performed using SPSS program V.21.0 (SPSS Inc., Chicago, IL, USA). A $P$ value $<0.05$ will be considered statistically significant. For cases in which we do not finish all the treatment, we select intention-to-treat (ITT) analysis to avoid the effects of crossover and drop-out. Measured data will be expressed as mean $\pm \mathrm{SD}(\bar{x} \pm \mathrm{s})$ if it obeys a normal distribution or approximate normal distribution. Median (interquartile range [IQR]) will be used if the data do not obey the normal distribution and count data will be expressed in terms of the number of cases. We will use chi-square tests for categorical data and twosample t-test or Wilcoxon rank sum test for continuous data, according to whether the data are normally distributed. The variance analysis will be performed for the difference between the two groups and within the group. The stratified analysis will be performed to control the confounding factor if necessary. Data analysis will be conducted by statisticians who are independent of the research team.

\section{Discussion}

Acupuncture therapy is a major component of TCM and is increasingly widely used because it is non-invasive and has fewer side effects [30-32]. The drugs for ovulation induction may lead to adverse reactions such as OHSS and multiple pregnancy. Compared with previous studies, we combined the TCM therapy with scientific and rigorous experimental design to explore the effect of MA on infertile women with PCOS. The selection of acupoints and herbs is based on the syndrome differentiation and the period of the female menstrual cycle in order to adequately demonstrate the dialectical treatment of TCM.

In terms of the treatment period, the acupuncture treatment starts on the third day of menstruation to the third day of ovulation; therefore, acupuncture can be fully utilized to promote the development and discharge of follicles and can avoid the risk if the patient is pregnant.

The evidence for long-term efficacy of acupuncture in patients with PCOS is not sufficient. However, a neurological study [33] showed that though SA and placebo were as effective as TA in terms of reduction of symptoms and objective physiological outcomes, verum acupuncture was superior to SA in producing improvements in both peripheral and brain neurophysiological outcomes. Pregnancy is the ultimate indicator of infertility treatment and insulin resistance (IR) is a pathological condition in which cells fail to respond normally to the insulin [34-36]. Of people with PCOS, $50-80 \%$ may have IR at some level [37]. IR is closely related to the pathogenesis of PCOS [38]. Therefore, we select the pregnancy rate, ovulation rate, sex hormones, and IRI as indicators of efficiency of the trial. We expect to discover that MA can be more helpful for promoting follicular development, adjusting menstrual cycle, and increasing pregnancy rate in infertile women with PCOS.

\section{Trial status}

At the time of initial manuscript submission, recruitment had already started (23 December 2017), but it has not been completed. The last patient is expected to be included in the study in February 2019.

\section{Additional file}

Additional file 1: SPIRIT 2013 Checklist: Recommended items to address in a clinical trial protocol and related documents. (PDF $130 \mathrm{~kb}$ )

\section{Abbreviations \\ CAM: Complementary and Alternative Medicine; HOMA-IR: Insulin resistance index; IR: Insulin resistance; IRI: Insulin Resistance Index; OHSS: Ovarian hyper- stimulation syndrome; PCOS: Polycystic ovary syndrome; RCT: Randomized controlled trial; SA: Sham acupuncture; TA: True acupuncture; TCM: Traditional Chinese Medicine}

\section{Acknowledgments}

Thanks to all the patients, nurses, and professionals in Shuguang Hospital who provided valuable input to the trial. We would also like to thank the staff of the Clinical Research Center, Shanghai Shuguang Hospital, for their advice and support throughout the trial process. 


\section{Authors' contributions}

The idea of the trial was conceived by $L Z$. XY, HD, and KC were involved in research design and primary writing of study protocol. QW was involved in the design and writing of the manuscript. ZH and WS will responsible for the recruitment and treatment of patients. XS, YZ, and $Y Y$ were involved in the trial design, writing, editing, and approval of the final manuscript. All authors read and approved the final manuscript.

\section{Funding}

This trial is supported by innovation funds of Shanghai Municipal Education Commission, China.

\section{Availability of data and materials}

The data that support the findings of this study will be available from authors but restrictions apply to the availability of these data, which will be used under license for the current study, and so are not publicly available. Data will be available from the authors upon reasonable request.

\section{Ethics approval and consent to participate}

The ethics committee has been approved by Institutional Review Board of Shuguang hospital affiliated with Shanghai University of TCM (22 December 2017, approval no. 2017-569-52-01). Written informed consent will be obtained from all participants.

\section{Consent for publication}

Not applicable. Results will be presented at relevant national and international conferences as well as being published in peer-reviewed journals.

\section{Competing interests}

The authors declare that they have no competing interests.

\section{Author details}

'Acupuncture \& Tuina School, Shanghai University of Traditional Chinese Medicine, Shanghai 201203, China. ${ }^{2}$ Shanghai Pudong New District Hospital of Traditional Chinese Medicine, Shanghai 201200, China. ${ }^{3}$ Shuguang Hospital, Shanghai University of Traditional Chinese Medicine, Shanghai 201203, China.

Received: 16 September 2018 Accepted: 19 August 2019 Published online: 11 September 2019

\section{References}

1. Group E. Revised 2003 consensus on diagnostic criteria and long-term health risks related to polycystic ovary syndrome (PCOS). Hum Reprod. 2004;81:19-25

2. Yildiz BO, Bozdag G, Yapici Z, Esinler I, Yarali H. Prevalence, phenotype and cardiometabolic risk of polycystic ovary syndrome under different diagnostic criteria. Hum Reprod. 2012;27:3067-73.

3. Gorry A, White DM, Franks S. Infertility in polycystic ovary syndrome: Focus on low-dose gonadotropin treatment. Endocrine. 2006;30:27-33.

4. Baird DT, Balen A, Escobar-Morreale HF, Evers JLH, Fauser BCJM, Franks S, et al. Health and fertility in World Health Organization group 2 anovulatory women. Hum Reprod Update. 2012:18:586-99.

5. Group TTEA. Consensus on infertility treatment related to polycystic ovary syndrome. Fertil Steril. 2008;89:505-22.

6. Kousta E, White DM, Franks S. Modern use of clomiphene citrate in induction of ovulation. Hum Reprod Update. 1997;3:359-65.

7. Tulandi T, Martin J, Al-Fadhli R, Kabli N, Forman R, Hitkari J, et al. Congenital malformations among 911 newborns conceived after infertility treatment with letrozole or clomiphene citrate. Fertil Steril. 2006;85:1761-5.

8. Reefhuis J, Honein MA, Schieve LA, Rasmussen SA. Use of clomiphene citrate and birth defects, National Birth Defects Prevention Study, 1997-2005. Hum Reprod. 2011;26:451-7.

9. Zhou J, Qu F. Treating gynaecological disorders with traditional Chinese medicine: A review. Afr J Tradit Complement Altern Med. 2009;6:494-517.

10. Cochrane S, Smith CA, Possamai-Inesedy A, Bensoussan A. Acupuncture and women's health: An overview of the role of acupuncture and its clinical management in women's reproductive health. Int J Women's Health. 2014;6:313-25.
11. Smith JF, Eisenberg ML, Millstein SG, Nachtigall RD, Shindel AW. The use of complementary and alternative fertility treatment in couples seeking fertility care: Data from a prospective cohort in the United States. Fertil Steril. 2010;93:2169-74.

12. Xia JF, Inagaki Y, Zhang JF, Wang L, Song PP. Chinese medicine as complementary therapy for female infertility. Chin J Integr Med. 2017;23:245-52.

13. Ma QW, Tan Y. Effectiveness of co-treatment with traditional Chinese medicine and letrozole for polycystic ovary syndrome: A meta-analysis. J Integr Med. 2017;15:95-101.

14. Yin Y, Zhang Y, Zhang H, Jiang D, Guo G. Clinical therapeutic effects of acupuncture combined with Chinese herbal medicine on infertility of polycystic ovary syndrome in the patients with ovulation induction with letrozole. Zhongguo Zhen Jiu. 2018;38:27-32.

15. Miner SA, Robins S, Zhu YJ, Keeren K, Gu V. Evidence for the use of complementary and alternative medicines during fertility treatment: $A$ scoping review. BMC Complement Altern Med. 2018;18:158.

16. $\mathrm{Xu} \mathrm{J,} \mathrm{Zuo} \mathrm{Y.} \mathrm{Efficacy} \mathrm{of} \mathrm{acupuncture} \mathrm{as} \mathrm{adjunctive} \mathrm{treatment} \mathrm{on} \mathrm{infertility}$ patients with polycystic ovary syndrome. Zhongguo Zhen Jiu. 2018;38:358-61.

17. Yang D, Zhao M, Tan J. Effect of polycystic ovary syndrome treated with the periodic therapy of acupuncture. Zhongquo Zhen Jiu. 2017;37:825-9.

18. Lim DC, Chen W, Cheng LN, Xue CC, Wong FW, O'Sullivan AJ, et al. Acupuncture for polycystic ovarian syndrome. Cochrane Database Syst Rev. 2011;96:D7689.

19. Zhu M. The medical classic of the yellow emperor. Beijing: Foreign Languages Press; 2001

20. Pastore LM, Williams CD, Jenkins J, Patrie $\int$. True and sham acupuncture produced similar frequency of ovulation and improved LH to FSH ratios in women with polycystic ovary syndrome. J Clin Endocrinol Metab. 2011;96:3143-50.

21. Wu XK, Stener-Victorin E, Kuang HY, Ma HL, Gao JS, Xie LZ, et al. Effect of acupuncture and clomiphene in chinese women with polycystic ovary syndrome: A randomized clinical trial. JAMA. 2017; 317:2502-14

22. Fan AY, Sherman G, Guanhu Y. Is acupuncture ineffective in treating infertility associated with polycystic ovary syndrome? A reanalysis of data on the clinical trial by WU Xiaoke's team published on JAMA. Zhongguo Zhen Jiu. 2017;37:1337.

23. Yang J, Liu Y, Huang J, Xu J, You X, Lin Q, et al. Acupuncture and Chinese medicine of artificial cycle therapy for insulin resistance of polycystic ovary syndrome with phlegm damp type and its mechanism. Zhongguo Zhen Jiu. 2017;37:1163-8.

24. Min Z, Hong-yan Y, Xiao-yun W, Jing-jing D, Jian L. Randomized controlled study of disease-syndrome combination therapy for anovulatory infertility. J Guangzhou Univ Tradit Chin Med. 2017;34(6):815-8.

25. Xie X, Gou WL. Obstetrics and gynecology. Beijing: People's Medical Publishing House; 2013.

26. Ji Z, Bai-xiao Z, Lixing L. Acupuncture and moxibustion. Beijing: People's Medical Publishing House; 2014.

27. Liu B, Xu H, Ma R, Mo Q, Yan S, Liu Z. Effect of blinding with a new pragmatic placebo needle: A randomized controlled crossover study. Medicine (Baltimore). 2014;93:e200

28. Jiang M, Lu C, Zhang C, Yang J, Tan Y, Lu A, et al. Syndrome differentiation in modern research of traditional Chinese medicine. J Ethnopharmacol. 2012;140:634-42.

29. Defronzo RA, Tobin JD, Andres R. Glucose clamp technique: A method for quantifying insulin secretion and resistance. Am J Physiol. 1979;237:E214.

30. Tan L, Tong $Y$, Sze SC, Xu M, Shi $Y$, Song XY, et al. Chinese herbal medicine for infertility with anovulation: A systematic review. J Altern Complement Med. 2012;18:1087-100

31. Ried K, Stuart K. Efficacy of Traditional Chinese Herbal Medicine in the management of female infertility: A systematic review. Complement Ther Med. 2011;19:319-31.

32. Liao WT, Chiang JH, Li CJ, Lee MT, Su CC. Investigation on the use of traditional Chinese medicine for polycystic ovary syndrome in a nationwide prescription database in taiwan. J Clin Med. 2018;7:179.

33. Maeda Y, Kim H, Kettner N, Kim J, Cina S, Malatesta C, et al. Rewiring the primary somatosensory cortex in carpal tunnel syndrome with acupuncture. Brain. 2017:140:914-27.

34. Schiavo L, Scalera G, Barbarisi A. Sleeve gastrectomy to treat concomitant polycystic ovary syndrome, insulin and leptin resistance in a 27-years morbidly obese woman unresponsive to insulin-sensitizing drugs: A 3-year follow-up. Int J Surg Case Rep. 2015;17:36-8.

35. Muscogiuri G, Colao A, Orio F. Insulin-Mediated diseases: Adrenal mass and polycystic ovary syndrome. Trends Endocrinol Metab. 2015; 26:512-4. 
36. Huang R, Yue J, Sun Y, Zheng J, Tao T, Li S, et al. Increased serum chemerin concentrations in patients with polycystic ovary syndrome: Relationship between insulin resistance and ovarian volume. Clin Chim Acta. 2015;450:366-9.

37. Teede H, Deeks A, Moran L. Polycystic ovary syndrome: A complex condition with psychological, reproductive and metabolic manifestations that impacts on health across the lifespan. BMC Med. 2010;8:41.

38. Sun L, Lv H, Wei W, Zhang D, Guan Y. Angiotensin-converting enzyme D/I and plasminogen activator inhibitor-1 4G/5G gene polymorphisms are associated with increased risk of spontaneous abortions in polycystic ovarian syndrome. J Endocrinol Investig. 2010;33:77-82.

\section{Publisher's Note}

Springer Nature remains neutral with regard to jurisdictional claims in published maps and institutional affiliations.

Ready to submit your research? Choose BMC and benefit from:

- fast, convenient online submission

- thorough peer review by experienced researchers in your field

- rapid publication on acceptance

- support for research data, including large and complex data types

- gold Open Access which fosters wider collaboration and increased citations

- maximum visibility for your research: over $100 \mathrm{M}$ website views per year

At $\mathrm{BMC}$, research is always in progress.

Learn more biomedcentral.com/submissions 